\title{
Klinik für Dermatologie, Venerologie und Allergologie der Universität des Saarlandes: Fortsetzung der tradierten Linie mit neuen wissenschaftlichen und klinischen Akzenten
}

\author{
Clinic of Dermatology, Venerology, and Allergology at the University of the Saarland: Keeping up Time Traditions, \\ Adding New Scientific and Clinical Key Aspects
}

Autor

Institut

\section{T. Vogt}

Direktor der Klinik für Dermatologie, Venerologie und Allergologie, Universitätsklinikum des Saarlandes

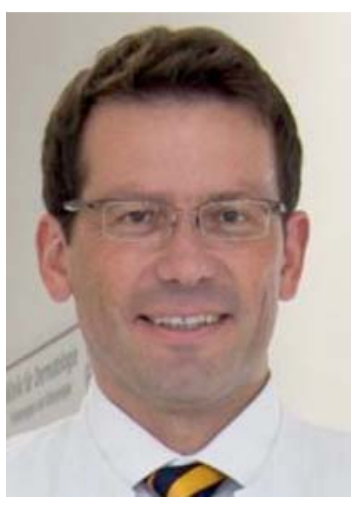

Prof. Dr. med. Thomas Vogt
Bibliografie

DOI http://dx.doi.org/

10.1055/s-0030-1256119

Akt Dermatol 2011; 37:

1-2 ๑ Georg Thieme

Verlag KG Stuttgart · New York ISSN 0340-2541

\footnotetext{
Korrespondenzadresse

Univ-Prof. Dr. med. Thomas Vogt

Direktor der Klinik für Dermatologie, Venerologie und Allergologie, Universitätsklinikum des Saarlandes Kirrbergerstr. 1 - Gebäude 18 66421 Homburg/Saar thomas.vogt@ uniklinikum-saarland.de
}

Die wechselvolle Geschichte der Hautklinik im äußersten Westen der Republik, nur wenige Autominuten entfernt von unseren Nachbarn in Frankreich und Luxemburg, liest sich wie ein spannendes Stück Zeitgeschichte. Die Abhandlung zur Geschichte unserer Klinik, verfasst von meiner/em Kollegin/en Sigrid Psaier und Knuth Rass in dieser Ausgabe der aktuellen Dermatologie, kann ich Ihnen daher nur empfehlen. Einen Besuch sollte Ihnen diese Klinik bei Gelegenheit immer Wert sein, schon wegen der bemerkenswerten kulinarisch-önologischen Begleitumstände in dieser Genießerregion.

Heute hat die Hautklinik der Universität des Saarlandes eine moderne und umfassende Infrastruktur. Es wird das gesamte Leistungsspektrum der konservativen und operativen Dermatologie angeboten mit Schwerpunkten in der operativen Dermatologie inklusive der Phlebochirurgie mit sämtlichen offenen und endoluminalen Verfahren, der Dermato-Onkologie mit dem vollen Spektrum der medikamentösen Tumortherapie, multimodalen Chemotherapie und der extrakorporalen Fotopherese. Homburg ist seit Jahren als vergleichsweise kleine Einheit mit führend bei der Rekrutierung von onkologischen Patienten in nationale und internationale Therapiestudien, vielleicht auch eines der wenigen Zentren mit ganzwöchiger, ganztägiger Tumorsprechstunde mit allen Angeboten der Diagnostik und der ambulanten Chemo- und Immuntherapien. Zwei großzügige moderne Operationssäle plus Eingriffsraum in räumlicher Einheit mit einem jüngst modernst ausgestatteten, leistungsfähigen Dermatohistologie-Labor, diktatfreie SAP-gestützte Dokumentation der OP-Berichte, z.T. auch der Histobefunde, straff prozessorientierte Organisation, Umstellung vieler Eingriffe von der Allgemeinanästhesie auf Tumeszenzverfahren u.a. ermöglichen die fortgesetzte Reduktion der Liegezeiten operativer Patienten und die über Jahre fortgesetzte Steigerung stationärer Fälle und ope- rativer Eingriffe ( $\bullet$ Tab. 1). Die Zahl der Histologien überschreitet 2010 erstmals die hausinterne „magische“ Marke von 5000, zudem kamen aus einer Kooperation mit dem LNS Luxemburg seit Mai 2010 erstmals bis zum heutigen Tag knapp 6000 weitere Einsendehistologien dazu. Für neue Laborgeräte und moderne Mikroskope inklusive Fotodokumentation in der Histologie wurden 2010 knapp 100000 Euro investiert. Die operative Verantwortlichkeit tragen neben dem Direktor OA Dr. Knuth Rass, OÄ Dr. Cornelia S. L. Müller und OA PD Dr. Alexander Rösch. Sowohl Frau Müller als auch der neue Direktor führen die Zusatzbezeichnung Dermatohistologie bzw. zusätzlich das European Board Certificate für Dermatopathologie.

Historisch gewachsen sind die Schwerpunkte Fotodermatologie - Vitamin D-Forschung, repräsentiert durch den ltd. OA Prof. Dr. Jörg Reichrath, ganz genauso die Allergologie-Berufdermatologie mit dem Ehrgeiz, ein saarländisches Handekzemzentrum zu gründen sowie ein interdisziplinäres Allergiezentrum gemeinsam mit den Kliniken für Pädiatrie, Pulmologie, Arbeitsmedizin, HNO und Gastroenterologie vertreten durch Ö̈ PD Dr. Claudia Pföhler.

Wissenschaftlich ist es ein vorrangiges Ziel der Hautklinik, den Einfluss des endokrinen Vitamin D-Systems u.a. auf die Hautkrebsentstehung (siehe Minireview in dieser Ausgabe) zu erforschen. Im Mai 2011 wird es in Homburg einen zweiten internationalen Kongress zur Vitamin DForschung, gefördert durch die Deutsche Forschungsgemeinschaft, DFG, geben (4th International Symposium: Vitamin D and Analogs in Cancer Prevention and Therapy 20-21 Mai 2011, Schlossberg Hotel Homburg). Auch wurden aktuell zwei neue Forschungsvorhaben definiert und befinden sich in der Antragsphase. Im Zuge der Neuberufung stehen erhebliche investive Mittel der Universität und Fakultät zur Verfügung neben bereits eingeworbenen Drittmitteln (ca. 350000 
Tab. 1 Auszüge Leistungszahlen $01-11 / 2010$.

$\begin{array}{lr}\text { Ambulante Behandlungsfälle } & 12094 \\ \text { Stationäre Behandlungsfälle } & 2021 \\ \text { Patienten in der Hautkrebssprechstunde } & 1465 \\ \text { Exzisionen von erkranktem Gewebe } & 1185 \\ \text { Lappenplastiken } & 613 \\ \text { Abgeschlossene Lichttherapien } & 266 \\ \text { Operative Varizenentfernung } & 183\end{array}$

EUR 2010-12), um die wissenschaftliche Laborgeräteausstattung in der Hautklinik weiter abzurunden. Die Stärke des Standorts ist jedoch neben einem räumlich und technisch zureichenden Labor in der Hautklinik vor allem die Vernetzung und unkomplizierte Zusammenarbeit mit den Partnern in anderen Kliniken, dem Jose Carreras Zentrum, dem Institut für experimentelle Chirurgie, den Instituten der theoretischen und vorklinischen Fächer mit einem SFB-Schwerpunkt im Bereich der Erforschung membrannaher Prozesse und der Ionenkanäle. Die Hautklinik verfolgt auch hier in Kooperation innovative Projekte im Bereich der Erforschung der Calcium-Ionenkanäle in Melanomzellen.

Unter diesen günstigen Voraussetzungen war es bereits im zu Ende gehenden Jahr möglich, aus den USA, vom renommierten Wistar Institut in Philadelphia, Herrn PD Dr. Alexander Rösch an die Hautklinik der Universität des Saarlandes zu holen und auch ein ambitioniertes DFG-gefördertes Projekt zur Melanomforschung (siehe Minireview zur Melanomstammzelle in dieser Ausgabe) zu initiieren.
So ist das Team aktuell auf fünf Oberärzte und 12 Assistenten sowie vier Gastärzte bzw. Gastwissenschaftler angewachsen. Drei Kollegen befinden sich auf „Habilitationskurs“, die Lehre wurde in diesem Jahr exzellent evaluiert, zahlreiche Doktoranden rekrutiert, nahezu alle PJ-Studenten der Hautklinik wollen auch hier ihre Ausbildung fortsetzen und fühlen sich wohl. Die Assistenten waren in den Weiterbildungsgesprächen durchweg motiviert und sehr zufrieden. Der Nachwuchs an Jungdermatologen scheint so für die Region auf einem guten Weg zu sein.

Die Interaktion mit den niedergelassenen Kollegen und Zuweisern wird ebenfalls belebt durch attraktive Fortbildungsangebote (drei Veranstaltungen allein im laufenden Jahr), andererseits durch intensivierte Zusammenarbeit in der Weiterbildung junger Kolleginnen und Kollegen mit den Praxen im Lande sowie gemeinsame prozessorientierte Arbeit zur Verbesserung der Abläufe gerade im Bereich der Versorgung von Hautkrebspatienten.

Unser Qualitätsziel ist eine ganzheitliche, an den individuellen Bedürfnissen der Patienten angepasste Diagnostik und Therapie von Hauterkrankungen. Zufriedene Patienten werden eine unabdingbare Grundlage für den Erfolg einer Klinik bleiben. Daher sind in das Behandlungskonzept stets auch berufliche und sozialmedizinische Aspekte eng eingebunden. Die harmonische und reibungslos vernetzte Zusammenarbeit mit den niedergelassenen Kollegen ist auch unter diesem Aspekt eine wichtige Voraussetzung und immer ein Gewinn für unsere Patienten und unsere aktuelle Dermatologie.

Mit besten Grüßen aus Homburg

Ihr

Thomas Vogt 